\title{
Intuitionistic fuzzy Dirichlet problem
}

\author{
S. Melliani, I. Bakhadach, M. Elomari and L. S. Chadli \\ Department of Mathematics, Sultan Moulay Slimane University \\ BP 523, 23000, Beni Mellal, Morocco \\ e-mail: saidmelliani@gmail.com
}

Received: 7 October $2018 \quad$ Revised: 21 November $2018 \quad$ Accepted: 24 November 2018

\begin{abstract}
In the present paper, a new definition of intuitionistic fuzzy derivative is presented, which a generalization of fuzzy fractional derivative and is compatible with the "crisp" definition of fractional derivative. We prove some properties concerning this definition. Finally, the existence of Dirichlet problem is proven.
\end{abstract}

Keywords: Hukuhara difference, Generalized intuitionistic fuzzy derivative, Intuitionistic fuzzy Caputo fractional derivative, Intuitionistic fuzzy Dirichlet problem.

2010 Mathematics Subject Classification: 03E72, 34Gxx.

\section{Introduction}

The present paper gives a methode of solving the following intuitionistic fuzzy problem by means of the intuitionistic fuzzy Laplace transform.

$$
\left\{\begin{array}{l}
\left({ }_{g H}^{c} D^{\beta} x(t)\right)=f(t, x(t)), \quad t \in I=[0, T] \\
{ }_{g H}^{c} D^{\beta-1} x(0)=x_{0} \in \mathbb{F}_{1}
\end{array},\right.
$$

where $0<\beta \leq 1$, the operator ${ }_{g H}^{c} D^{\beta}$ denotes the Caputo fractional generalized derivative of order $\beta, f: I \times \mathbb{F}_{1}(\mathbb{R}) \longrightarrow \mathbb{F}_{1}(\mathbb{R})$.

Fractional calculus is a mathematical branch investigating the properties of derivatives and integrals of non-integer orders. It applies in modelling of many physical and chemical processes and in engineering [3]. Podlubny [12] and Kilbas et al [9] gave the idea of fractional calculus and consider Riemann-Liouville differentiability to solve FFDEs. Agarwal et al. [1] proposed the concept of solutions for fractional differential equations with uncertainty. 
Intuitionistic fuzzy sets (IFS) were first formulated by Atanassov (see [4,5]). An IFS is a generalization of fuzzy sets introduced by Zadeh [14]. In fuzzy sets, the membership value, $(\mu(x))$, of $x \in X$ (called the universe) is just a single real number, usually in $[0,1]$ and the nonmembership of $x$ is taken as $(1-\mu(x))$. But for intuitionistic fuzzy sets the membership value $(\mu(x))$ as well as the non-membership value $(\nu(x))$ should be taken into account for describing any $x$ in $X$ such that the sum of membership and non-membership is less than or equal to 1 . Thus, an IFS is expressed by an ordered pair of real numbers $(\mu(x), \nu(x))$ and $\pi(x)=1-\mu(x)-\nu(x)$ is called the hesitancy. There have been several attempts to quantify the uncertainty associated with fuzzy sets as well as with intuitionistic fuzzy sets [7]. The authors in [8] studied the solution concept of fractional differential equations with intuitionistic fuzzy initial data under generalized fuzzy Caputo derivative. In [11] Melliani et al. introduced the extension of Hukuhara difference in the intuitionistic fuzzy case. Allahviranloo, Armand and Gouyandeh in [2] solve the fuzzy fractional differential equations under generalized fuzzy Caputo derivative. From this last paper, we introduce in this paper the concept of generalized intuitionistic fuzzy Caputo derivative and we will build our ideas and properties in order to solve the fractional equation by means of the Laplace transform, which is the one of the interesting transforms used for solving intuitionistic fuzzy differential equations. Using this transform allows reduction of the problem. The advantage of intuitionistic fuzzy Laplace transform is to solve the problem directly without determining a general solution.

The present paper investigate the analytic solution of the following problem

$$
\left\{\begin{array}{l}
\Delta u(x)=f, \quad x \in] a, b[ \\
u(a)=A \in \mathcal{F} T, \\
u(b)=B \in \mathcal{F} T,
\end{array}\right.
$$

where $\mathcal{F} T$ is the set of all intuitionistic fuzzy triangular numbers, and $f$ is a function defined from ]$a, b[$ into $\mathcal{F} T$.

This paper is organized as follows. After this introduction, we recall in Section 2 some concept concerning the fuzzy metric space and generalized Hukuhara's difference, which inspired the method of build a fuzzy Banach space. The generalized differentiability takes place in the later section. We will presented the main result in Section 5 and we end our work by an example in order to illustrate our results.

\section{Preliminaries}

In this section we will present some definitions and properties, which we will build our work upon.

Definition 1. [10] The set of all intuitionistic fuzzy numbers is given by

$$
\mathbb{F}_{1}=\mathbb{F}_{1}(\mathbb{R})=\left\{\langle u, v\rangle: \mathbb{R} \longrightarrow[0,1]^{2}, \quad 0 \leq u+v \leq 1\right\}
$$

with the following conditions: 
1. For each $\langle u, v\rangle \in \mathbb{F}_{1}$ is normal, i.e, $\exists x_{0}, x_{1} \in \mathbb{R}$, such that $u\left(x_{0}\right)=1$ and $v\left(x_{1}\right)=1$.

2. For each $\langle u, v\rangle \in \mathbb{F}_{1}$ is a convex intuitionistic set, i.e., $u$ is fuzzy convex and $v$ is fuzzy concave.

3. For each $\langle u, v\rangle \in \mathbb{F}_{1}$, $u$ is lower continuous and $v$ is upper continuous.

4. $\overline{\{x \in \mathbb{R}, v(x) \leq \alpha\}}$ is bounded.

Definition 2. [10] For $\alpha \in[0,1]$, we define the upper and lower $t$-cut by

$$
\begin{aligned}
& {[\langle u, v\rangle]_{\alpha}=\{x \in \mathbb{R}: u(x) \geq \alpha\},} \\
& {[\langle u, v\rangle]^{\alpha}=\{x \in \mathbb{R}: v(x) \leq 1-\alpha\} .}
\end{aligned}
$$

Definition 3. The intuitionistic fuzzy zero is an intuitionistic fuzzy set defined by

$$
\widetilde{0}(x)=\left\{\begin{array}{ll}
(1,0) & x=0 \\
(0,1) & x \neq 0
\end{array} .\right.
$$

Proposition 1. [10] We can write

$$
\begin{aligned}
& {[\langle u, v\rangle]_{\alpha}=\left[[\langle u, v\rangle]_{l}^{+}(\alpha),[\langle u, v\rangle]_{r}^{+}(\alpha)\right],} \\
& {[\langle u, v\rangle]^{\alpha}=\left[[\langle u, v\rangle]_{l}^{-}(\alpha),[\langle u, v\rangle]_{r}^{-}(\alpha)\right] .}
\end{aligned}
$$

Remark 1. In the fuzzy case, we can write $[\langle u, v\rangle]_{\alpha}=[u]^{\alpha}$ and $[\langle u, v\rangle]^{\alpha}=[1-v]^{\alpha}$.

Proposition 2. [10] For all $\langle u, v\rangle,\left\langle u^{\prime}, v^{\prime}\right\rangle \in \mathbb{F}_{1}$, we have

$$
\langle u, v\rangle=\left\langle u^{\prime}, v\right\rangle \Longleftrightarrow\left\{\begin{array}{ll}
{[\langle u, v\rangle]_{\alpha}=\left[\left\langle u^{\prime}, v\right\rangle\right]_{\alpha}} & \\
{[\langle u, v\rangle]^{\alpha}=\left[\left\langle u^{\prime}, v\right\rangle\right]^{\alpha}} &
\end{array} \quad \forall t \in[0,1] .\right.
$$

We define two operations on $\mathbb{F}_{1}$ by

$$
\begin{gathered}
\langle u, v\rangle \oplus\left\langle u^{\prime}, v\right\rangle=\left\langle u \vee v, u^{\prime} \wedge v^{\prime}\right\rangle, \quad \forall\langle u, v\rangle,\left\langle u^{\prime}, v\right\rangle \in \mathbb{F}_{1}, \\
\lambda\langle u, v\rangle=\langle\lambda u, \lambda v\rangle, \quad \forall \lambda \in \mathbb{R}, \forall\langle u, v\rangle \in \mathbb{F}_{1} .
\end{gathered}
$$

According to Zadeh extension, we have

$$
\begin{gathered}
{\left[\langle u, v\rangle \oplus\left\langle u^{\prime}, v\right\rangle\right]_{\alpha}=[\langle u, v\rangle]_{\alpha}+\left[\left\langle u^{\prime}, v\right\rangle\right]_{\alpha},} \\
{\left[\langle u, v\rangle \oplus\left\langle u^{\prime}, v\right\rangle\right]^{\alpha}=[\langle u, v\rangle]^{\alpha}+\left[\left\langle u^{\prime}, v\right\rangle\right]^{\alpha},} \\
{[\lambda\langle u, v\rangle]_{\alpha}=\lambda[\langle u, v\rangle]_{\alpha},} \\
{[\lambda\langle u, v\rangle]^{\alpha}=\lambda[\langle u, v\rangle]^{\alpha} .}
\end{gathered}
$$


Theorem 1. [10] Let $\mathcal{M}=\left\{M_{\alpha}, M^{\alpha}, \alpha \in[0,1]\right\}$ be a family of subsets in $\mathbb{R}$ satisfying the following conditions

1. $\alpha \leq s \Longrightarrow M_{s} \subset M_{\alpha}$ and $M^{s} \subset M^{\alpha}$, for each $\alpha, s \in[0,1]$.

2. $M_{\alpha}$ and $M_{s}$ are nonempty compact convex sets in $\mathbb{R}$, for each $\alpha \in[0,1]$.

3. For any non-decreasing sequence $\alpha_{i} \longrightarrow \alpha$ on $[0,1]$, we have for each $\alpha \in[0,1] . M_{\alpha}=\bigcap_{i} M_{\alpha_{i}}$ and $M^{\alpha}=\bigcap_{i} M^{\alpha_{i}}$.

We define $u$ and $v$ by

$$
\begin{gathered}
u(x)=\left\{\begin{array}{cc}
0, & x \notin M_{0} \\
\sup _{\alpha \in[0,1]} M_{\alpha} & x \in M_{0},
\end{array}\right. \\
v(x)=\left\{\begin{array}{cc}
1, & x \notin M^{0} \\
1-\sup _{\alpha \in[0,1]} M_{\alpha} & x \in M^{0} .
\end{array}\right.
\end{gathered}
$$

Then

$$
\langle u, v\rangle \in \mathbb{F}_{1}
$$

with $M_{\alpha}=[\langle u, v\rangle]_{\alpha}$ and $M^{\alpha}=[\langle u, v\rangle]^{\alpha}$.

Remark 2. [10]

1. The family $\left\{[\langle u, v\rangle]_{\alpha},[\langle u, v\rangle]^{\alpha}, \alpha \in[0,1]\right\}$ satisfying conditions 1 . - 3. of the previous theorem.

2. For all $\alpha \in[0,1]$,

$$
[\langle u, v\rangle]_{\alpha} \subset[\langle u, v\rangle]^{\alpha}
$$

Theorem 2. [10] On $\mathbb{F}_{1}$ we can define the metric

$$
\begin{aligned}
& d_{\infty}((u, v),(z, w))=\frac{1}{4} \sup _{0<\alpha \leq 1}\left|[(u, v)]_{r}^{+}(\alpha)-[(z, w)]_{r}^{+}(\alpha)\right| \\
& +\frac{1}{4} \sup _{0<\alpha \leq 1}\left|[(u, v)]_{l}^{+}(\alpha)-[(z, w)]_{l}^{+}(\alpha)\right|+\frac{1}{4} \sup _{0<\alpha \leq 1}\left|[(u, v)]_{r}^{-}(\alpha)-[(z, w)]_{r}^{-}(\alpha)\right| \\
& +\frac{1}{4} \sup _{0<\alpha \leq 1}\left|[(u, v)]_{l}^{-}(\alpha)-[(z, w)]_{l}^{-}(\alpha)\right|
\end{aligned}
$$

and

$$
\begin{aligned}
& d_{p}\left(\langle u, v\rangle,\left\langle u^{\prime}, v\right\rangle\right)=\left(\frac{1}{4} \int_{0}^{1}\left|[\langle u, v\rangle]_{l}^{+}(\alpha)-\left[\left\langle u^{\prime}, v\right\rangle\right]_{l}^{+}(\alpha)\right| d t\right. \\
&+\frac{1}{4} \int_{0}^{1}\left|[\langle u, v\rangle]_{r}^{+}(\alpha)-\left[\left\langle u^{\prime}, v\right\rangle\right]_{r}^{+}(\alpha)\right| d t+ \frac{1}{4} \int_{0}^{1}\left|[\langle u, v\rangle]_{l}^{-}(\alpha)-\left[\left\langle u^{\prime}, v\right\rangle\right]_{l}^{-}(\alpha)\right| d t \\
&\left.+\frac{1}{4} \int_{0}^{1}\left|[\langle u, v\rangle]_{r}^{-}(\alpha)-\left[\left\langle u^{\prime}, v\right\rangle\right]_{r}^{-}(\alpha)\right| d t\right)^{\frac{1}{p}}
\end{aligned}
$$

for $p \in[1, \infty)$. We have that $\left(\boldsymbol{I F}_{1}, d_{p}\right)$ is a complete metric space. 


\subsection{The generalized Hukuhara derivative of an intuitionistic fuzzy-valued function}

The concept of intuitionistic fuzzy Hukuhara difference was introduced by the authors in [11]. In this paper we will give the definition of generalized Hukuhara difference between two intuitionistic fuzzy numbers.

Definition 4. The generalized Hukuhara difference of two fuzzy numbers $\langle u, v\rangle$ and $\left\langle u^{\prime}, v\right\rangle \in \mathbb{F}_{1}$ is defined as follows

$$
\langle u, v\rangle-_{g H}\left\langle u^{\prime}, v\right\rangle=\langle z, w\rangle \Longleftrightarrow\langle u, v\rangle=\left\langle u^{\prime}, v\right\rangle \oplus\langle z, w\rangle .
$$

Note that the $\alpha$-level representation of a fuzzy-valued function $f:(0, T] \longrightarrow \mathbb{F}_{1}$ expressed by

$[f]_{\alpha}=\left[f_{\alpha, l}, f_{\alpha, r}\right]$ and $[f]^{\alpha}=\left[f^{\alpha, l}, f^{\alpha, r}\right]$.

Definition 5. The generalized Hukuhara derivative of an intuitionistic fuzzy-valued function $f$ : $(0, T] \longrightarrow I F_{1}$ at $t_{0}$ is defined as

$$
f_{g H}^{\prime}\left(t_{0}\right)=\lim _{t \rightarrow t_{0}} \frac{f(t)-{ }_{g H} f\left(t_{0}\right)}{t-t_{0}}
$$

if $f_{g H}^{\prime}\left(t_{0}\right) \in I F_{1}$, we say that $f$ is generalized Hukuhara differentiable at $t_{0}$

Also we say that $f$ is $[(i)-g H]$-differentiable at $t_{0}$ if

$$
\left\{\begin{array}{l}
\left(f_{g H}^{\prime}\right)_{\alpha}=\left[\left(f_{\alpha, l}\right)^{\prime},\left(f_{\alpha, r}\right)^{\prime}\right] \\
\left(f_{g H}^{\prime}\right)^{\alpha}=\left[\left(f^{\alpha, l}\right)^{\prime},\left(f^{\alpha, r}\right)^{\prime}\right]
\end{array}\right.
$$

and that $f$ is $[(i i)-g H]$-differentiable at $t_{0}$ if

$$
\left\{\begin{array}{l}
\left(f_{g H}^{\prime}\right)_{\alpha}=\left[\left(f_{\alpha, r}\right)^{\prime},\left(f_{\alpha, l}\right)^{\prime}\right] \\
\left(f_{g H}^{\prime}\right)^{\alpha}=\left[\left(f^{\alpha, r}\right)^{\prime},\left(f^{\alpha, l}\right)^{\prime}\right]
\end{array}\right.
$$

We can define the generalized derivative of higher order by

$$
\left\{\begin{array}{l}
f^{(0)}=f \\
f_{g H}^{(n)}=\left(f^{(n-1)}\right)_{g H}^{\prime}, \quad \forall n \in \mathbb{N}
\end{array} .\right.
$$

Definition 6. Let $f:(0, T) \longrightarrow \mathbb{F}_{1}$. We say that $f$ of class $\mathcal{C}^{m}, m \in \mathbb{N}$, if $f_{\text {gh }}^{(m)}$ exists and continues, with respect to metric $d_{\infty}$.

Now if the $\alpha$-levels of $f:(0, T] \longrightarrow \mathbb{F}_{1}$, are given by $[f]_{\alpha}=\left[f_{\alpha, l}, f_{\alpha, r}\right]$ and $[f]^{\alpha}=\left[f^{\alpha, l}, f^{\alpha, r}\right]$ and $f_{\alpha, l}, f_{\alpha, r}, f^{\alpha, l}, f^{\alpha, r}$ are Riemann integrable on $(0, T]$.

Since the family

$$
\left\{\left[f_{\alpha, l}, f_{\alpha, r}\right], \quad\left[f^{\alpha, l}, f^{\alpha, r}\right]\right\}
$$


built an intuitionistic fuzzy element and the integral preserves the monotony, then by Theorem 1 the family

$$
\left\{\left[\int_{(0, T]} f_{\alpha, l}, \int_{(0, T]} f_{\alpha, r}\right],\left[\int_{(0, T]} f^{\alpha, l}, \int_{(0, T]} f^{\alpha, r}\right]\right\}
$$

defines an intuitionistic fuzzy element, which is the integral of $f$ on $(0, T]$, we denote $\int_{(0, T]} f$.

Definition 7. Let $f:(0, T] \longrightarrow \boldsymbol{F}_{1}$ be a intuitionistic fuzzy-valued function. We say that $f$ is integrable on $(0, T]$ if $f_{\alpha, l}, f_{\alpha, r}, f^{\alpha, l}, f^{\alpha, r}$ defined in the previous are integrable on $(0, T]$

Remark 3. By the same method we can extended this definition in order to define the impropre intuitionistic fuzzy Riemann integral.

\section{Intuitionistic fuzzy generalized caputo-derivative}

Let $f:(0, T] \longrightarrow \mathbb{F}_{1}$ be an intuitionistic fuzzy-valued integrable function on $(0, T]$, and $\delta \in(m-1, m]$ and $m \in \mathbb{N}^{*}$.

The lower and upper $\alpha$-levels of $f$ are defined as follows :

$[f]_{\alpha}=\left[f_{\alpha, l}, f_{\alpha, r}\right]$ and $[f]^{\alpha}=\left[f^{\alpha, l}, f^{\alpha, r}\right]$, where $f_{\alpha, l}, f_{\alpha, r}, f^{\alpha, l}, f^{\alpha, r} \in \mathcal{C}^{m}((0, T])$.

We set

$$
M_{\alpha}=\left[\frac{1}{\Gamma(\delta)} \int_{0}^{t}(t-s)^{\delta-m-1}\left(f_{\alpha, l}\right)^{(m)}(s), \frac{1}{\Gamma(\delta)} \int_{0}^{t}(t-s)^{\delta-m-1}\left(f_{\alpha, r}\right)^{(m)}(s)\right]
$$

and

$$
M^{\alpha}=\left[\frac{1}{\Gamma(\delta)} \int_{0}^{t}(t-s)^{\delta-m-1}\left(f^{\alpha, l}\right)^{(m)}(s), \frac{1}{\Gamma(\delta)} \int_{0}^{t}(t-s)^{\delta-m-1}\left(f^{\alpha, r}\right)^{(m)}(s)\right] .
$$

Proposition 3. The family $\left\{M_{\beta}, M^{\beta}, \beta \in[0,1]\right\}$ defines an intuitionistic fuzzy element.

Proof. Just use Theorem 2.

Definition 8. The intuitionistic fuzzy preceding item is called the generalized Caputo derivative of $f$, we denote $D^{\alpha} f$.

We say that $f$ is ${ }^{c f}[(i)-g H]$-differentiable at $t_{0}$ if

$$
\left[{ }_{g H} D^{\delta} f\right]_{\alpha}=\left[D^{\delta} f_{\alpha, l}, D^{\delta} f_{\alpha, r}\right], \quad\left[{ }_{g H} D^{\delta} f\right]^{\alpha}=\left[D^{\delta} f^{\alpha, l}, D^{\delta} f^{\alpha, r}\right]
$$

and that $f$ is ${ }^{c f}[(i i)-g H]$-differentiable at $t_{0}$ if

$$
\left[{ }_{g H} D^{\delta} f\right]_{\alpha}=\left[D^{\delta} f_{\alpha, r}, D^{\delta} f_{\alpha, l}\right], \quad\left[{ }_{g H} D^{\delta} f\right]^{\alpha}=\left[D^{\delta} f^{\alpha, r}, D^{\delta} f^{\alpha, l}\right] .
$$

As in the previuos definition, we will give the definition of intuitionistic fuzzy fractional Riemann-Liouville integral. If the $\alpha$-levels of $f:(0, T] \longrightarrow \mathbb{F}_{1}$ are given by $[f]_{\alpha}=\left[f_{\alpha, l}, f_{\alpha, r}\right]$ and $[f]^{\alpha}=\left[f^{\alpha, l}, f^{\alpha, r}\right]$ and $f_{\alpha, l}, f_{\alpha, r}, f^{\alpha, l}, f^{\alpha, r}$ are Riemann integrable on $(0, T]$.

Since the family

$$
\left\{\left[f_{\alpha, l}, f_{\alpha, r}\right],\left[f^{\alpha, l}, f^{\alpha, r}\right]\right\}
$$


represents an intuitionistic fuzzy element and the integral preserves the monotony, then we have by Theorem 1 the family

$$
\left\{\mathcal{A}_{\alpha}, \mathcal{A}^{\alpha}, \alpha \in[0,1]\right\}
$$

where

$$
\mathcal{A}_{\alpha}=\left[\frac{1}{\Gamma(\delta)} \int_{(0, t)}(t-s)^{\delta-1} f_{\alpha, l}(s), \frac{1}{\Gamma(\delta)} \int_{(0, t)}(t-s)^{\delta-1} f_{\alpha, r}(s)\right]
$$

and

$$
\mathcal{A}^{\alpha}=\left[\frac{1}{\Gamma(\delta)} \int_{(0, t)}(t-s)^{\delta-1} f^{\alpha, l}(s), \frac{1}{\Gamma(\delta)} \int_{(0, t)}(t-s)^{\delta-1} f^{\alpha, r}(s)\right]
$$

define an intuitionistic fuzzy element, which is the Riemann-Liouville fractional integral of $f$ on $(0, T)$, we denote $\frac{1}{\Gamma(\delta)} \int_{(0, t)}(t-s)^{\delta-1} f(s) d s$.

Definition 9. [9] Let $f:(0, T] \longrightarrow \mathbb{F}_{1}$ be a continuous function. The Riemann-Liouville fractional integral of $f$ on $(0, T)$ is defined as

$$
I^{\delta} f(t)=\frac{1}{\Gamma(\delta)} \int_{(0, t)}(t-s)^{\delta-1} f(s) d s
$$

where $\delta \in(m-1, m)$

\section{Intuitionistic fuzzy generalized Hukuhara partial differentiation}

Throught this paper we denote $\mathcal{T} F$ the sets of all triangular intuitionistic fuzzy numbers. We use the same proof as Bede in [6], we can show that if $\langle u, v\rangle,\left\langle u^{\prime}, v\right\rangle \in \mathcal{T} F$, then the difference $\langle u, v\rangle-{ }_{g}\left\langle u^{\prime}, v\right\rangle$ always exists in $\mathcal{T} F$ and $\langle u, v\rangle-{ }_{g}\left\langle u^{\prime}, v\right\rangle=(-1)\left(\langle u, v\rangle-{ }_{g}\left\langle u^{\prime}, v\right\rangle\right)$. Thus we get $\left(\mathcal{F} T,\|\cdot\|_{p}\right)$ is a Banach space. In this section $f: \mathbb{D} \subset \mathbb{R} \times \mathbb{R}^{+} \rightarrow \mathcal{F} T$ is called the two variable fuzzy-valued function. The parametric representation of the fuzzy-valued function fis expressed by $f\left(x, t, \alpha^{-}\right)=\left[\underline{f}\left(x, t, \alpha^{-}\right), \bar{f}\left(x, t, \alpha^{-}\right)\right]$and $f\left(x, t, \alpha^{-}\right)=\left[\underline{f}\left(x, t, \alpha^{+}\right), \bar{f}\left(x, t, \alpha^{+}\right)\right]$.

Definition 10. Let $f: \mathbb{D} \subset \mathbb{R} \times \mathbb{R}^{+} \rightarrow \mathbb{F}_{1}$ and $\left(x_{0}, t_{0}\right) \in \mathbb{D}$. Then first generalized Hukuhara partial derivative $([\mathrm{gH}-\mathrm{p}]$-derivative for short) of $f$ with respect to variables $x, t$ are the functions $\partial_{x_{g H}} f\left(x_{0}, t_{0}\right)$ and $\partial_{t_{g H}} f\left(x_{0}, t_{0}\right)$ given by

$$
\lim _{h \rightarrow 0}\left\|\frac{f\left(x_{0}+h, t_{0}\right)-{ }_{g} f\left(x_{0}, t_{0}\right)}{h}-{ }_{g} \partial_{x_{g H}} f\left(x_{0}, t_{0}\right)\right\|=0
$$

and

$$
\lim _{h \rightarrow 0}\left\|\frac{f\left(x_{0}, t_{0}+h\right)-{ }_{g} f\left(x_{0}, t_{0}\right)}{h}, \partial_{x_{g H}} f\left(x_{0}, t_{0}\right)\right\|=0,
$$

provided that $\partial_{x_{g H}} f\left(x_{0}, t_{0}\right), \partial_{t_{g H}} f\left(x_{0}, t_{0}\right) \in \mathcal{T} F$.

Definition 11. Let $f(x, t): \mathbb{D} \rightarrow \mathcal{T} F,\left(x_{0}, t_{0}\right) \in \mathbb{D} . \quad \underline{f}\left(x, t ; \alpha^{+}\right), \bar{f}\left(x, t ; \alpha^{+}\right), \underline{f}\left(x, t ; \alpha^{-}\right)$, $\bar{f}\left(x, t ; \alpha^{-}\right)$both partial differentiable w.r.t. $t$ at $\left(x_{0}, t_{0}\right)$. We say that 
- (1). $f(x, t)$ is $[(i)-p]$-differentiable w.r.t. t at $\left(x_{0}, t_{0}\right)$ if

$$
\begin{aligned}
& {\left[\partial_{t_{i, g H}} f\left(x_{0}, t_{0}\right)\right]^{\alpha}=\left[\partial_{t} \underline{f}\left(x_{0}, t_{0} ; \alpha^{-}\right), \partial_{t} \bar{f}\left(x_{0}, t_{0} ; \alpha^{-}\right)\right]} \\
& {\left[\partial_{t_{i, g H}} f\left(x_{0}, t_{0}\right)\right]_{\alpha}=\left[\partial_{t} \underline{f}\left(x_{0}, t_{0} ; \alpha^{+}\right), \partial_{t} \bar{f}\left(x_{0}, t_{0} ; \alpha^{+}\right)\right]}
\end{aligned}
$$

- (2). $f(x, t)$ is $[(i i)-p]$-differentiable w.r.t. t at $\left(x_{0}, t_{0}\right)$ if

$$
\begin{aligned}
& {\left[\partial_{t_{i i, g H}} f\left(x_{0}, t_{0}\right)\right]^{\alpha}=\left[\partial_{t} \bar{f}\left(x_{0}, t_{0} ; \alpha^{-}\right), \partial_{t} \underline{f}\left(x_{0}, t_{0} ; \alpha^{-}\right)\right]} \\
& {\left[\partial_{t_{i i, g H}} f\left(x_{0}, t_{0}\right)\right]_{\alpha}=\left[\partial_{t} \bar{f}\left(x_{0}, t_{0} ; \alpha^{+}\right), \partial_{t} \underline{f}\left(x_{0}, t_{0} ; \alpha^{+}\right)\right]}
\end{aligned}
$$

Inspired from [13], we present the following definition.

Definition 12. $f: \mathbb{R} \times \mathbb{R}^{+} \rightarrow \mathcal{T} F$. We say that the function $t=h(x)$, is switching boundary for the differentiability of $f(x, t)$ with respect to $t$, if for all $x$ belonging to the domain of $h$ and for all $t \in \mathbb{R}^{+}$, there exist points $t_{0}<t_{1}<t_{2}$ such that

1. at $\left(x, t_{1}\right)(1)$ holds while (2) does not hold and at $\left(x, t_{2}\right)(2)$ holds and (1) does not hold, or

2. at $\left(x, t_{1}\right)(2)$ holds while (1) does not hold and at $\left(x, t_{2}\right)(1)$ holds and (2) does not hold.

Theorem 3. Consider $f: \mathbb{R} \times \mathbb{R}^{+} \rightarrow \mathcal{T} F$ and $u: \mathbb{R} \rightarrow E^{1}$ are fuzzy-valued functions such that $u(x ; \alpha)=[\underline{u}(x ; \alpha), \bar{u}(x ; \alpha)]$. Suppose that $h: \mathbb{R} \rightarrow \mathbb{R}$ and $p: \mathbb{R} \times \mathbb{R}^{+} \rightarrow \mathbb{R}^{+}$is a differentiable function w.r.t. $t$ and

$$
\partial_{t} p(x, t)= \begin{cases}\partial_{t} p(x, t) \geq 0, & h_{1}(t)<x<h_{2}(t) ; \\ \partial_{t} p(x, t)<0, & h_{2}(t)<x<h_{3}(t)\end{cases}
$$

and $f(x, t)=p(x, t) u(x)$. Then $\partial_{t_{g H}} f(x, t)$ exists and

$$
\partial_{t_{g H}} p(x, t)= \begin{cases}\partial_{t_{i, g H}} p(x, t) \geq 0, & h_{1}(t)<x<h_{2}(t) ; \\ \partial_{t_{i i, g H}} p(x, t)<0, & h_{2}(t)<x<h_{3}(t)\end{cases}
$$

In fact, the function $h_{2}(t)$ is switching boundary type 1 for differentiability of $f(x, t)$ with respect to $t$.

Proof. Since $p$ is valued in $\mathbb{R}^{+}$then we can set $[f(x, t ; \alpha)]^{\alpha}=p(x, t)\left[\underline{u}\left(x ; \alpha^{-}\right), \bar{u}\left(x ; \alpha^{-}\right)\right]$, $[f(x, t ; \alpha)]_{\alpha}=p(x, t)\left[\underline{u}\left(x ; \alpha^{+}\right), \bar{u}\left(x ; \alpha^{+}\right)\right]$, which implies that

$$
\left[\partial_{t_{g H}}\right]=\partial_{t} p(x, t)[\underline{u}(x ; \alpha), \bar{u}(x ; \alpha)]
$$

If $h_{1}(t)<x<h_{2}(t)$ then

$$
\begin{aligned}
& {\left[\partial_{t_{g H}}\right]^{\alpha}=\left[\partial_{t} p(x, t) \underline{u}\left(x ; \alpha^{-}\right), \partial_{t} p(x, t) \bar{u}\left(x ; \alpha^{-}\right)\right]} \\
& {\left[\partial_{t_{g H}}\right]_{\alpha}=\left[\partial_{t} p(x, t) \underline{u}\left(x ; \alpha^{+}\right), \partial_{t} p(x, t) \bar{u}\left(x ; \alpha^{+}\right)\right]}
\end{aligned}
$$


then $f(x, t)$ is [ $(i)$-differentiable] by report at $t$. In the same manner, if $h_{2}(t)<x<h_{3}(t)$ we get

$$
\begin{aligned}
& {\left[\partial_{t_{g H}}\right]^{\alpha}=\left[\partial_{t} p(x, t) \bar{u}\left(x ; \alpha^{-}\right), \partial_{t} p(x, t) \underline{u}\left(x ; \alpha^{-}\right)\right]} \\
& {\left[\partial_{t_{g H}}\right]_{\alpha}=\left[\partial_{t} p(x, t) \bar{u}\left(x ; \alpha^{+}\right), \partial_{t} p(x, t) \underline{u}\left(x ; \alpha^{+}\right)\right]}
\end{aligned}
$$

thus $f(x, t)$ is $[(i i)$-differentiable] with respect to the second variable $t$.

\section{Intuitionistic fuzzy Dirichlet problem in one dimension}

In this section, we will investigade the following problem

$$
\left\{\begin{array}{l}
\Delta u(x)=f, \quad x \in[a, b] \\
u(a)=A \in \mathcal{T} F \\
u(b)=B \in \mathcal{T} F
\end{array}\right.
$$

where $f$ is a suitable function.

We introduce the auxiliary function $v$ verifying $u^{\prime}=v$ and $v^{\prime}=f$.

Proposition 4. A mapping $u:[a, b] \rightarrow \mathcal{T} F$ is a solution of (3) if only if $v$ is a solution of the following problem:

$$
\left\{\begin{array}{l}
v^{\prime}(x)=f \quad x \in[a, b] \\
u(a)=A \in \mathcal{T} F \\
u(b)=B \in \mathcal{T} F
\end{array}\right.
$$

if $u$ is (i)-diff and $v$ is (i)-diff or if $u$ is (ii)-diff and $v$ is (ii)-diff.

And A mapping $u:[a, b] \rightarrow \mathcal{T} F$ is a solution of (3) if only if $v$ is a solution of the following problem:

$$
\left\{\begin{array}{l}
v^{\prime}(x)=-{ }_{g H} f \quad x \in[a, b] \\
u(a)=A \in \mathcal{T} F \\
u(b)=B \in \mathcal{T} F
\end{array}\right.
$$

if $u$ is (i)-diff and $v$ is (ii)-diff or if $u$ is (ii)-diff and $v$ is (i)-diff.

Proof. Since $u, v$ and $f$ are at values in $\mathbb{F}_{1}$, we put $u=\left\langle u_{1}, u_{2}\right\rangle, v=\left\langle v_{1}, v_{2}\right\rangle$ and $f=\left\langle f_{1}, f_{2}\right\rangle$. We will discuss four cases.

1. If $u$ is $(i)$-diff and $v$ is $(i)$-diff.

In this case we have

$$
\left[\left\langle u_{1}^{\prime}, u_{2}^{\prime}\right\rangle\right]^{\alpha}=\left[\left\langle v_{1}, v_{2}\right\rangle\right]^{\alpha} \text { and }\left[\left\langle u_{1}^{\prime}, u_{2}^{\prime}\right\rangle\right]_{\alpha}=\left[\left\langle v_{1}, v_{2}\right\rangle\right]_{\alpha},
$$

which implies that

$$
\left[\left\langle v_{1}^{\prime}, v_{2}^{\prime}\right\rangle\right]^{\alpha}=\left[\left\langle f_{1}, f_{2}\right\rangle\right]^{\alpha}, \quad \text { and }\left[\left\langle v_{1}^{\prime}, v_{2}^{\prime}\right\rangle\right]_{\alpha}=\left[\left\langle f_{1}, f_{2}\right\rangle\right]_{\alpha} .
$$

Using Theorem 2, we get $v^{\prime}=f$. 
2. If $u$ is $(i i)$-diff and $v$ is $(i i)$-diff.

In this case we have

$$
\left[\left\langle u_{1}^{\prime}, u_{2}^{\prime}\right\rangle\right]^{\alpha}=\left[\left\langle v_{2}, v_{1}\right\rangle\right]^{\alpha}, \quad \text { and } \quad\left[\left\langle u_{1}^{\prime}, u_{2}^{\prime}\right\rangle\right]_{\alpha}=\left[\left\langle v_{2}, v_{1}\right\rangle\right]_{\alpha}
$$

Which implies that

$$
\left[\left\langle v_{1}^{\prime}, v_{2}^{\prime}\right\rangle\right]^{\alpha}=\left[\left\langle u_{1}^{\prime \prime}, u_{2}^{\prime \prime}\right\rangle\right]^{\alpha}=\left[\left\langle f_{1}, f_{2}\right\rangle\right]^{\alpha}
$$

in the same

$$
\left[\left\langle v_{1}^{\prime}, v_{2}^{\prime}\right\rangle\right]_{\alpha}=\left[\left\langle f_{1}, f_{2}\right\rangle\right]_{\alpha}
$$

Using Theorem 2 we get $v^{\prime}=f$.

3. If $u$ is $(i)$-diff and $v$ is $(i i)$-diff.

In this case we have

$$
\left[\left\langle u_{1}^{\prime}, u_{2}^{\prime}\right\rangle\right]^{\alpha}=\left[\left\langle v_{1}, v_{2}\right\rangle\right]^{\alpha}
$$

and

$$
\left[\left\langle u_{1}^{\prime}, u_{2}^{\prime}\right\rangle\right]_{\alpha}=\left[\left\langle v_{1}, v_{2}\right\rangle\right]_{\alpha}
$$

which implies that

$$
\left[\left\langle v_{1}^{\prime}, v_{2}^{\prime}\right\rangle\right]^{\alpha}=\left[\left\langle u_{2}^{\prime \prime}, u_{1}^{\prime \prime}\right\rangle\right]^{\alpha}=\left[\left\langle f_{2}, f_{1}\right\rangle\right]^{\alpha}
$$

in the same

$$
\left[\left\langle v_{1}^{\prime}, v_{2}^{\prime}\right\rangle\right]_{\alpha}=\left[\left\langle f_{2}, f_{1}\right\rangle\right]_{\alpha} .
$$

Also by Theorem 2 we get $v^{\prime}=-{ }_{g H} f$.

4. If $u$ is $(i i)$-diff and $v$ is $(i)$-diff.

In this case we have

$$
\left[\left\langle u_{1}^{\prime}, u_{2}^{\prime}\right\rangle\right]^{\alpha}=\left[\left\langle v_{2}, v_{1}\right\rangle\right]^{\alpha},
$$

and

$$
\left[\left\langle u_{1}^{\prime}, u_{2}^{\prime}\right\rangle\right]_{\alpha}=\left[\left\langle v_{2}, v_{1}\right\rangle\right]_{\alpha},
$$

which implies that

$$
\left[\left\langle v_{1}^{\prime}, v_{2}^{\prime}\right\rangle\right]^{\alpha}=\left[\left\langle u_{2}^{\prime \prime}, u_{1}^{\prime \prime}\right\rangle\right]^{\alpha}=\left[\left\langle f_{2}, f_{1}\right\rangle\right]^{\alpha}
$$

in the same

$$
\left[\left\langle v_{1}^{\prime}, v_{2}^{\prime}\right\rangle\right]_{\alpha}=\left[\left\langle f_{2}, f_{1}\right\rangle\right]_{\alpha} .
$$

Also by Theorem 2 we get $v^{\prime}=-{ }_{g H} f$.

Finally we obtain $v^{\prime}=f$ if $u$ is $(i)$-diff and $v$ is $(i)$-diff or if $u$ is (ii)-diff and $v$ is (ii)-diff, and $v^{\prime}={ }_{g H} f$ if $u$ is $(i)$-diff and $v$ is $(i i)$-diff or if $u$ is $(i i)$-diff and $v$ is $(i)$-diff.

Before we turn to the solution, we will give a property of the map $v$. 
Lemma 1. $v$ is a continuous function.

Proof. We have

$$
\left\{\begin{array}{l}
v(t)=v(a)+\int_{a}^{t} f(s) d s \\
x(t)=x(a)+\int_{a}^{t} v(s) d s
\end{array}\right.
$$

Thus

$$
v(a)=\frac{1}{b-a}\left\{B-{ }_{g H} \int_{a}^{b} \int_{a}^{t} f(\tau) d \tau d t\right\}
$$

Since

$$
\begin{aligned}
B_{1}\left(\alpha^{-}\right)-{ }_{g H} \int_{a}^{b} \int_{a}^{t} \underline{f}\left(\tau, \alpha^{-}\right) d \tau d t & =\int_{a}^{b} \frac{B_{1}\left(\alpha^{-}\right)}{b-a}-{ }_{g H} \int_{a}^{t} \underline{f}\left(\tau, \alpha^{-}\right) d \tau d t \\
& =\int_{a}^{b}\left\{\left[\frac{B_{1}\left(\alpha^{-}\right)}{(b-a)(t-a)}-{ }_{g H} \int_{a}^{t} \underline{f}\left(\tau, \alpha^{-}\right)\right] d \tau\right\} d t
\end{aligned}
$$

The function $\left[\frac{B_{1}(\alpha)}{(b-a)(t-a)}-{ }_{g H} \int_{a}^{t} \underline{f}\left(\tau, \alpha^{-}\right) d \tau\right]$ is monotone increasing on $I$ with respect to $\alpha$, In the same manner, the we get

$$
\begin{aligned}
B_{2}\left(\alpha^{+}\right)-{ }_{g H} \int_{a}^{b} \int_{a}^{t} \bar{f}\left(\tau, \alpha^{+}\right) d \tau d t & =\int_{a}^{b} \frac{B_{2}\left(\alpha^{+}\right)}{b-a}-{ }_{g H} \int_{a}^{t} \bar{f}\left(\tau, \alpha^{+}\right) d \tau d t \\
& =\int_{a}^{b}\left\{\left[\frac{B_{2}\left(\alpha^{+}\right)}{(b-a)(t-a)}-{ }_{g H} \int_{a}^{t} \bar{f}\left(\tau, \alpha^{+}\right)\right] d \tau\right\} d t
\end{aligned}
$$

The function $\left[\frac{B_{2}\left(\alpha^{+}\right)}{(b-a)(t-a)}-{ }_{g H} \int_{a}^{t} \bar{f}\left(\tau, \alpha^{+}\right) d \tau\right]$ is monotone decreasing on $I$ with respect to $\alpha$.

By Theorem 1 we get the following property.

$$
\frac{B(\alpha)}{(b-a)(t-a)}-{ }_{g H} \int_{a}^{t} \bar{f}(\tau) d \tau \in E^{1} .
$$

Thus $v$ is a continuous derivable map on $[a, b]$.

Theorem 4. The solution of (3) is given by

$$
x(t)=A+(t-a) v(a)+\int_{a}^{t} \int_{a}^{s} f(\tau) d \tau d t
$$

if $x$ is (i)-diff and $x^{\prime}$ is (i)-diff or if $x$ is (ii)-diff and $x^{\prime}$ is (ii)-diff.

$$
x(t)=A+(t-a) v(a)-\int_{a}^{t} \int_{a}^{s}-{ }_{g H} f(\tau) d \tau d t
$$

if $x$ is (i)-diff and $x^{\prime}$ is (ii)-diff or if $x$ is (ii)-diff and $x^{\prime}$ is (i)-diff.

Proof. First $x$ is $(i)$-diff and $x^{\prime}$ is $(i i)$-diff or if $x$ is $(i i)$-diff and $x^{\prime}$ is $(i)$-diff.

We put

$$
\phi(t)=A+(t-a) v(a)+\int_{a}^{t} \int_{a}^{s} f(\tau) d \tau d t .
$$


Since $t \geq a$, the map

$$
\alpha \rightarrow A_{1}(\alpha)+(t-a) \underline{v}\left(a, \alpha^{-}\right)+\int_{a}^{t} \int_{a}^{s} \underline{f}\left(\tau, \alpha^{-}\right) d \tau d s
$$

is monotone increasing on $I$ with respect to $\alpha$, and

$$
\alpha \rightarrow A_{2}(\alpha)+(t-a) \bar{v}\left(a, \alpha^{+}\right)+\int_{a}^{b} \int_{a}^{s} \bar{f}\left(\tau, \alpha^{+}\right) d \tau d s
$$

is monotone decreasing on $I$ with respect to $\alpha$.

So

$$
\phi(t) \in E^{1}, \quad \forall t \in[a, b] .
$$

We get also

$$
\phi^{\prime}(t)=v(a)+\int_{a}^{s} f(\tau) d \tau
$$

Thus,

$$
\phi^{\prime}(a)=v(a)
$$

and

$$
\phi^{\prime \prime}(t)=f(t),
$$

which implies that $\phi$ is a solution of (3). If $x$ is $(i)$-diff and $x^{\prime}$ is (ii)-diff.

By the same step we get

$$
\phi^{\prime \prime}=(-1) \otimes\left({ }_{g H} f\right)=f
$$

On the other hand, the problem

$$
\left\{\begin{array}{l}
\Delta x(t)=0, \quad t \in[a, b] \\
x(a)=x(b)=\widetilde{0}
\end{array}\right.
$$

has $\widetilde{0}$ as the unique solution.

Thus,

$$
x(t)=A+(t-a) v(a)+\int_{a}^{t} \int_{a}^{s} f(\tau) d \tau d t, \quad \forall t \in[a, b] .
$$

\section{References}

[1] Agarwal, R. P., Lakshmikantham, V., Nieto, J. J. (2010). On the concept of solution for fractional differential equations with uncertainty, Nonlinear Anal., 72(6), 2859-2862.

[2] Allahviranloo, T., Armand, A., \& Gouyandeh, Z. (2014). Fuzzy fractional differential equations under generalized fuzzy Caputo derivative, J. Intelligent and Fuzzy Systems, 26, 14811490. 
[3] Arara, A., Benchohra, M., Hamidi, N., \& Nieto, J. J. (2010). Fractional order differential equations on an unbounded domain, Nonlinear Anal., 72(2), 580-586.

[4] Atanassov, K. (1983). Intuitionistic fuzzy sets, VII ITKR Session, Sofia, 20-23 June 1983 (Deposed in Centr. Sci.-Techn. Library of the Bulg. Acad. of Sci., 1697/84) (in Bulgarian). Reprinted: Int. J. Bioautomation, 2016, 20(S1), S1-S6.

[5] Atanassov, K. (1986). Intuitionistic fuzzy sets, Fuzzy Sets and Systems, 20(1), 87-96.

[6] Bede, B., \& Stefanini, L. (2013). Generalized differentiability of fuzzy-valued functions, Fuzzy Sets and Systems, 230, 119-141.

[7] De Luca, A., \& Termini, S. (1972). A definition of non Probabilistic entropy in the setting of fuzzy theory, Information and Control, 20(4), 301-312.

[8] Elomari, M., Melliani, S., \& Chadli, L. S. (2017). Solution of intuitionistic fuzzy fractional differential equations. Annals of Fuzzy Mathematics and Informatics, 13(3), 379-391.

[9] Kilbas, A. A., Srivastava, H. M., \& Trujillo, J. J. (2006). Theory and applications of fractional differential equations, Amesterdam: Elsevier Science.

[10] Melliani, S., Elomari, M., Chadli, L. S. \& Ettoussi, R. (2015). Intuitionistic fuzzy metric space, Notes on Intuitionistic Fuzzy Sets, 21(1), 43-53.

[11] Melliani, S., Elomari, M., Chadli, L. S. \& Ettoussi, R. (2015). Extension of Hukuhara difference in intuitionistic fuzzy set theory, Notes on Intuitionistic Fuzzy Sets, 21 (4), 34-47.

[12] Podlubny, I. (1999). Fractional Differential Equations, Academic Press, San Diego.

[13] Stefanini, L., \& B. Bede, (2009). Generalized hukuhara differentiability of interval-valued functions and interval differential equations, Nonlinear Analysis: Theory, Methods and Applications 71(34), 1311-1328.

[14] Zadeh, L. A. (1965). Fuzzy sets, Information and Control, 8, 338-353 